Методологический подход к цифровизации и индустриализации развития региональных и муниципальных структур

на постковидном пространстве

\begin{tabular}{c} 
Роза Т. Тимакова \\
\hline \hline Ольга Т. Ергунова
\end{tabular}$\quad \begin{aligned} & \text { trt64@ mail.ru } \\
& \text { ergunova-olga@ ya.ru }\end{aligned}$
(D 0000-0002-1714-7784
Ключевые слова: индустриализация, цифровизация, цифровая экономика, региональное управление, муниципальное управление, интернет вещей, умный город, умный регион

\title{
Methodological approach to digitalization and industrialization of the development of regional and municipal structures in the post-Covid space
}

\begin{tabular}{|c|c|c|}
\hline $\begin{array}{l}\text { Roza T. Timakova }{ }^{1} \\
\text { Olga T. Ergunova }\end{array}$ & $\begin{array}{l}\text { trt64@mail.ru } \\
\text { ergunova-olga@ya.ru }\end{array}$ & $\begin{array}{l}\text { (D) } 0000-0002-4777-1465 \\
\text { (D) } 0000-0002-1714-7784\end{array}$ \\
\hline \multicolumn{3}{|c|}{1 Ural State Economic University, St. 8-e Marta/Narodnoy voli, 62/45, Ekaterinburg, 620144, Russia } \\
\hline $\begin{array}{l}\text { Abstract. The transformation of the economy in the context } \\
\text { mportance of digital preorientation of the development of re } \\
\text { 'smart" space that meets the level of development of society } \\
\text { oasis of the research is formed on the basis of content analysi } \\
\text { n digitalization and industrialization of management syster } \\
\text { ancertainty and transformation of economic conditions. The } \\
\text { egion" projects based on the introduction of information an } \\
\text { economic development of territories and improve the quality } \\
\text { nanagement methods, and social practices of digitalization } \\
\text { specialization, which implies the creation of the necessary } \\
\text { nformation society, as well as aimed at improving the efficier } \\
\text { and analysis.)Within the framework of the developed projects } \\
\text { at supporting small and medium-sized businesses most affec } \\
\text { pasis for different segments of the population including }\end{array}$ & $\begin{array}{l}\text { cordance with the concept of s } \\
\text { research and economic and sta } \\
\text { f subjects and municipalities } \\
\text { for implementing the initiati } \\
\text { nmunication technologies in } \\
\text { e of citizens are studied. Thes } \\
\text { industrial development of ter } \\
\text { astructure and the cultivation } \\
\text { f economic management proce } \\
\text { igitalization of the municipal } \\
\text { y the pandemic and the possi }\end{array}$ & $\begin{array}{l}\text { a entities, taking into a } \\
\text { ompetencies that are in } \\
\text { based on digital tools fol }\end{array}$ \\
\hline
\end{tabular}
Keywords: industrialization, digitalization, digital economy, regional government, municipal government, internet of things, smart city, smart region

Для цитирования

Тимакова Р.T., Ергунова О.Т. Методологический подход к цифровизации и индустриализации развития региональных и муниципальных структур на постковидном пространстве //Вестник ВГУИТ. 2020. Т. 82. № 4. С. 371-376. doi:10.20914/2310-1202-20204-371-376

(C) 2021, Тимакова P.T. и др. / Timakova R.T. et al
For citation

Timakova R.T., Ergunova O.T. Methodological approach to digitalization and industrialization of the development of regional and municipal structures in the post-Covid space. Vestnik VGUIT [Proceedings of VSUET]. 2020. vol. 82. no. 4. pp. 371-376. (in Russian). doi:10.20914/2310-1202-2020-4-371-376

This is an open access article distributed under the terms of the Creative Commons Attribution 4.0 International License 


\section{Введение}

Трансформация экономики в условиях распространения пандемии COVID-19 и социоэкономической нестабильности повышает значимость цифровизации и индустриализации развития региональных и муниципальных структур, усилив их влияние на формирование «smart» пространства, отвечающего уровню развития общества в соответствие с концепцией устойчивого развития. Ориентация на цифровую модель развития экономических процессов находит отражение в области нормативно-правового регулирования, внедрения новейших информационных и интерактивных технологий, больших данных, промышленного интернета вещей с учетом умной региональной специализации. Активное и поступательное развитие единого информационного пространства России становится направляющим трендом развития экономики, что определяет актуальность исследования особенностей и тенденций протекания процессов цифровизации и индустриализации на региональном и муниципальном уровне.

Цифровая трансформация экономики затрагивает в первую очередь сферу услуг, сосредоточенных в городах и являющихся неотьемлемыми частями обеспечения функционирования систем управления современных городов.

В настоящее время муниципальными органами власти совместно с региональными и государственными органами власти РФ ведутся активно работы по развитию единого информационного пространства России, которые реализуются через развитие местных (локальных) программ, которые являются составляющими этого пространства. Применение информационных технологий в управлении субъектами и муниципальными образованиями РФ возможно по нескольким направлениям:

- в работе с населением, т. е. использование различных «личных кабинетов» или наоборот общедоступных информационных площадок, как, например, «электронная регистратура»;

- при работе с государственными, региональными и муниципальными органами власти.

Вся эта совокупность различных информационных платформ, банков данных, базы данных, технологий представляет собой единое информационное пространство, которое работает и подчиняется общим принципам и правилам для взаимодействия с населением и удовлетворение их потребностей.

Становление и развитие концепции цифровизации объявлено одним из приоритетных направлений государственной политики через реализацию национальной программы «Цифровая экономика», принятой в 2017 году. Национальная программа в целом отвечает практике странчленов ОЭСР, где также реализуются стратегии и программы цифровизации экономик. В целях цифровизации муниципальных образований в РФ с 2019 по 2024 гг. реализуется проект «Умный город», направленный на повышение конкурентоспособности российских городов, данный проект базируется на ключевых принципах: комфортная и безопасная среда, повышение качества управления городскими ресурсами, ориентация на человека, экономическая эффективность, технологичность городской среды.

Представленные принципы проекта «Умный город» особенно важны в современных условиях неоиндустриализации и реиндустриализации, выражающихся «в смене поколений машин и оборудования в формате индустриального технологического уклада» [1].

Однако, имевшая место в 2019 году тенденция бюджетного невыполнения национальной программы «Цифровая экономика» (53,6\%), призванной стать основой для развития всех остальных федеральных программ, продолжилась в условиях ограничений, связанных с COVID-2019, показав худший результат по бюджетному исполнению за период январь-август 2020 года (17,8\%). Годовой бюджет нацпроекта на 2020 год составлял 151 млрд руб. при общем бюджете 1634,9 млрд руб. на 2018-2024 гг.

Соответственно существует необходимость оценки не только экономических параметров социально-экономического, институционального и другого порядка, определяющих эффективность реализации национального проекта, но и сопоставление качественных характеристик отечественной цифровизации муниципальных и региональных образований с зарубежными аналогами.

В Российской Федерации при достаточно стабильном положении в рейтинге стран мира по итогам 2017-2019 годов наблюдается значительное отставание от наиболее развитых экономик мира:

- 45 место в рейтинге Индекса развития ИКТ 2017 года (ICT Development Index, IDI) Международного союза электросвязи (МСЭ),

- 43 место в рейтинге Всемирного экономического форума о глобальной конкурентоспособности 2019 года по индексу глобальной конкурентоспособности,

- 38 место в международном рейтинге цифровой конкурентоспособности - IMD World Digital Competitiveness Ranking,

- 12 место среди 17 стран, не входящих в Европейский Союз, в рейтинге международного индекса цифровой экономики и общества (International Digital Economy and Society Index, I-DESI). 
Проанализировав данные рейтингов, к основным причинам отставания России в развитии цифровой экономики можно отнести: недостаточно благоприятная институциональная среда для введения инноваций, недостаточная эффективность правового регулирования, низкий объем инвестиций в развитие наукоемких технологий, а также невысокий уровень использования цифровых технологий субъектами и муниципальными образованиями. Различие в использовании цифровых сервисов в муниципальных образованиях РФ хорошо иллюстрируется динамикой проникновения локальных цифровых сервисов: доля горожан полностью обеспеченных цифровыми сервисами составляет $50,5 \%$, в то время 20,9\% их не получают; при этом удельный вес городов, полностью обеспеченных сервисом, составляет только 5\%; в 73,7\% городов отмечается полное отсутствие сервисных цифровых услуг (рисунок 1).

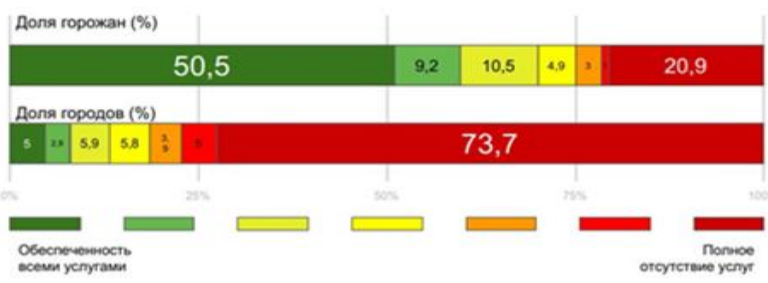

Рисунок 1. Проникновение локальных цифровых сервисов в города РФ

Figure 1. Digital services in cities of the Russian Federation

В соответствие с индексом цифровизации городского хозяйства «IQ городов» 2020 года лидерами рейтинга проекта «Умный город», реализующегося в рамках национальных проектов - «Жилье и городская среда» и «Цифровая экономика» среди мегаполисов стали: 1 место Москва (индекс 81,19) и далее Казань $(52,58)$, Санкт-Петербург (50,37), Нижний Новгород $(46,5)$ и Уфа (42,05), среди крупных городов: 1 местоХимки $(66,32)$ и далее Балашиха $(59,38)$, Тюмень (58,31), Подольск $(56,6)$ и Ставрополь $(45,69)$, среди больших городов лидируют: 1 место Реутов (индекс 71,35$)$ и далее Серпухов $(63,5)$, Электросталь $(61,88)$, Домодедово $(60,58)$ и Орехово-Зуево $(60,15)$. Среди административных центров и пилотных городов выделяется на 1-м месте Дубна $(72,48)$ и далее Ивантеевка $(62,18)$, Наро-Фоминск $(41,02)$, Гатчина $(39,19)$ и Елабуга $(38,04)$. Рейтинг муниципальных образований был составлен на основе базового уровня цифровизации городского хозяйства, эффективности управленческих решений согласно паспорту проекта «Умный город», существующего уровня технологического развития и перспективных направлений развития.
Формирование среды «Умного региона» основывается на глобальной тенденции внедрения концепции «Интернет вещей» (Internet of Things, IoT) в силу увеличения количества устройств, подключенных к глобальной сети, уже сейчас превышающих численность населения Земли почти в 3,5 раза. Данная трансформация и преобразование муниципальных образований в систему «Умного региона» позволит в будущем решить проблемы рационального использования энергетических ресурсов, обеспечения безопасности за счет создания эффективной инфраструктуры региона, города через внедрение системы связи и обмена через наличие единого интерфейса с множеством сервисов, в котором происходит обмен информацией в реальном времени.

К организационно-экономическим механизмам обеспечения цифровизации и индустриализации развития отечественных региональных и муниципальных структур в постковидном среднесрочном прогнозе можно отнести: развитие логистических транспортных и товарноматериальных потоков, использование энергосберегающих технологий; вопросы поддержки IP-телефонии и планирования расширения охвата сетей; развитие модели государственночастного партнерства в кредитно-финансовой и социальной сфере, в том числе в сфере образования за счет распространения открытого образовательного пространства $[2,3]$.

С точки зрения эффективности от внедрения цифровых технологий в пространство городов современные исследователи отмечают, что такие технологии как большие данные (Big Data), форсайт-аналитика, искусственный интеллект, машинное обучение (Machine Learning), промышленный интернет вещей (IIoT), облачные сервисы способствуют в повышении в разы уровня мобильности, социальных коммуникаций, привлекательности жилой среды в городах, удешевления решений в области производства, торговли и сферы услуг, жилищнокоммунального, транспортного, логистического хозяйства, энергетики, связи и др. [4-15].

Предложения зарубежных ученых направлены на расширение не только экономических, но и организационных механизмов обеспечения цифровизации и индустриализации развития регионов: создание союза представителей бизнеса, науки и власти; сотрудничество вузов и научно-исследовательских институтов для создания и реализации мероприятий по цифровизации и индустриализации развития региональной и муниципальной экономики; развитие межгосударственных наукоемких технологий и услуг. 


\section{Материалы и методы}

Материалом для исследования послужили информационные ресурсы административных органов субъектов и муниципальных образований, статистические материалы и научноисследовательская литература; применены общенаучные эмпирические методы исследования на основе сопоставимого анализа и интерпретации результатов исследования.

Основополагающие методологические и методические рекомендации по изучению и внедрению принципов цифровизационного и индустриального развития региональных и муниципальных структур рассматриваются в основном как совместное предпринимательство и бизнес-инкубаторы, инновационное развитие на основе количественной оценки и нахождения причинно-следственных связей; формирования теория продолжительности деловых циклов, что требует формализованного подхода в постковидном среднесрочном прогнозе.

\section{Результаты и обсуждение}

Принимая во внимание результаты исследования отечественных и зарубежных ученых, предлагается в рамках существующего методологического подхода к цифровизации и индустриального развития региональных и муниципальных структур в постковидном среднесрочном прогнозе (рисунок 2) в отличие от существующих методологий разработку следующих этапов: согласование стратегии с населением территории и формирование бюджета, анализ «провалов» цифровизации и индустриализации развития региональных и муниципальных структур в постковидном среднесрочном прогнозе и формирование бюджета территории на основе «провально-проектно - целевого подхода».

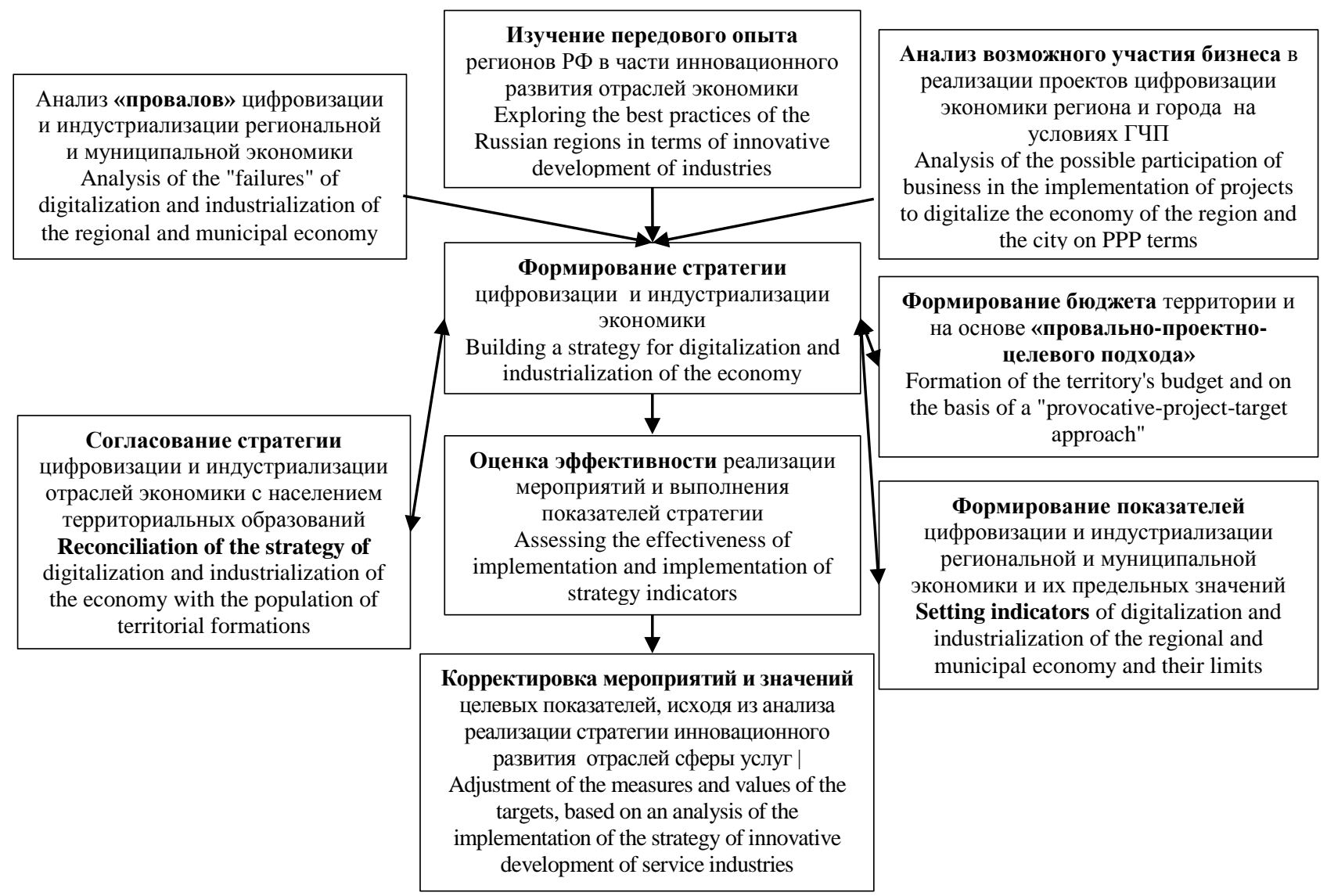

Рисунок 2. Разработка методологии цифровизационного и индустриального развития региональных и муниципальных структур в постковидном среднесрочном прогнозе

Figure 2. Development of a methodology for digitalization and industrial development of regional and municipal structures in the post-market medium-term forecast

При этом учитываются «провалы государства», проектируются мероприятия в рамках проекта с наибольшей эффективностью и существующей взаимосвязи мероприятий и целевых показателей (каждое мероприятие направлено на достижение определенного целевого показателя).
Разработанная методология позволит повысить эффективность формирования и реализации стратегии цифровизации и индустриализации развития региональных и муниципальных структур, ввести формирование бюджета территории, исходя из предлагаемого «провальнопроектно-целевого подхода». 
Концептуальным подходом при разработке стратегии по предлагаемым проектам в разрезе «регион-город» является адаптация отечественной экономики в условиях экономической турбулентности в целях решения вопросов формирования в регионах РФ пилотных площадок по разработке и внедрению высокотехнологичных решений для «Умных городов», повышения уровня и качества жизни населения, формирования безопасной и комфортной пространственной среды.

В рамках проектов предлагаются следующие мероприятия, в первую очередь направленные на социальную поддержку населения в условиях пандемии COVID-19:

1. «Транспорт»: развитие сети услуг метрополитена в мегаполисах; установление в общественном транспорте детских кресел (в целях безопасности детей); проведение регулярной санитарной обработки транспорта согласно требований Роспотребнадзора.

2. «Связь»: введение государством дотаций, обеспечивающие интернет-обслуживание учащихся общеобразовательных учреждений и лимитированную по тарифу сотовую связь; введение навигаторов «бейджиков» учащимся общеобразовательных учреждений и детям детских дошкольных учреждений.

3. «Оптовая и розничная торговля»: введение государством еженедельных дотационных пакетов для полноценного питания граждан, достигших пенсионного возраста, и детей до 18-летнего возраста.

4. «Кредитно-финансовая сфера»: расширение спектра инвестиционной поддержки со стороны бизнес-институтов развития представителям малого и среднего бизнеса;

5. «Страхование»: введение государством дотаций на страхование детей до 18 летнего возраста.

6. «Бытовые услуги»: введение на территориях схем оказания бытовых слуг пошаговой доступности.

7. «Культурно-рекреационные услуги»: организация и проведение культурных мероприятий в территориях на условии государственночастного партнерства.

8. «Услуги социального профиля - образование, здравоохранение, социальные услуги»: введение инновационных образовательных парков в территориях для обучения детей школьного возраста; введение государством дотаций на проведение бесплатных подготовительных курсов для детей дошкольного возраста; введение программы «непрерывного семейного образования» В дистанционном формате; создание проекта по обеспечению нуждающихся категорий бесплатным интернетом (школьники, студенты, пенсионеры); возможность получения бесплатного профессионального образования в открытом образовательном пространстве для социализации «особенных» людей (инклюзия) и профессиональной переподготовки работников предприятий отраслей экономики, наиболее пострадавших на фоне распространения коронавирусной инфекции.

Предложенные мероприятия позволят расширить спектр инновационного развития территории в масштабе «регион»- «город», повысить эффективность предоставления государственных и муниципальных услуг, увеличить эффективность расходования бюджетных средств, повысить качество стратегического планирования территории в постковидном среднесрочном прогнозе.

\section{Заключение}

В настоящее время вопрос формирования организационно-экономических механизмов обеспечения цифровизации и индустриализации развития региональных и муниципальных структур является важным и актуальным. В РФ формируется единое цифровое пространство через развитие местных (локальных) программ. В рамках национального проекта «Цифровая экономика» предлагается развитие «интернет вещей» для управления регионами и муниципальными образованиями. Трансформация муниципального и регионального управления на основе развития концепции построения «Умных городов и регионов» позволит не только решить внутренние проблемы развития городов РФ, предотвращая потенциальные угрозы, но и развивать инновации цифровизационного и индустриального развития региональных и муниципальных структур в постковидном среднесрочном прогнозе. Сформированные предложения по развитию проектов по отраслям деятельности имеют комплексный и целевой характер и направлены на эффективное развитие отраслей региональной и муниципальной экономики и социализацию разработанных решений.

\section{Литература}

1 Овчинников В.Н., Кетова Н.П. Системодополняющий эффект взаимодействия инновационного потенциала и институциональной среды региона // Экономика региона. 2016. Т. 12. № 2. С. 537-546. doi: 10.17059/2016-2-18

2 Timakova R.T., Maiseyenko A.V., Iliukhin R.V. Modern Aspects of Managing the Institutional Environment of Higher Education // Proceedings of the International Scientific Conference «Far East Con» (ISCFEC 2020). V. 128. P.1104-1112. doi:10.2991/aebmr.k. 200312.152

3 Тимакова Р.Т., Ильюхина Ю.В. Особенности открытого образовательного пространства Российской Федерации и Республики Беларусь в условиях цифровизации // Тенденции развития электронного образования в России и за рубежом: мат. I Междунар. науч.-практ. конф. 2020. C. 170-173. 
4 Головко О.И. Организационно-экономический механизм обеспечения инновационного развития организаций // Экономика и управление народным хозяйством. 2016. № 4 (137). С. 92-94.

5 Zhang M.L., Chen M.S. China's digital economy: Opportunities and risks. International Monetary Fund, 2019.

6 Крегель Д.А. Государственные механизмы стимулирования инновационной деятельности на железнодорожном транспорте // Вестник университета. 2018. № 2. С. 57-63.

7 Беспалова И.В. Трансформация инновационной модели в современных условиях структуры экономики.// Фундаментальные исследования. 2015. № 12 (2). С. 346-355.

8 Watanabe C. et al. Measuring GDP in the digital economy: Increasing dependence on uncaptured GDP // Technological Forecasting and Social Change. 2018. V. 137. P. 226-240.

9 Eleodor D. Big tech, big competition problem? // Quality-Access to Success. 2019. V. 20 (S3). P. 49-57. URL: https://search.proquest.com/openview/58271826c315d0334b7e9cc6e79a756a/1.pdf? pq-origsite=gscholar\&cbl=1046413

10 Kuladzhi T., Babkin A., Murtazaev S.A. Matrix tool for efficiency assessment of production of building materials and constructions in the digital economy // Advances in intelligent systems and computing. 2017. V. 692. P. 1333-1346. doi: 10.1007/978-3-319-70987-1_141

11 Blaginin V., Ergunova O., Pyankova S. Automobile transport infrastructure in the system of maintaining social well-being // Conference Title Abbreviation \& a Year Conference Title. 2018. P. 62-70.

12 Евсютин О.О., Кокурина А.С., Мещеряков Р.В. Обзор методов встраивания информации в цифровые объекты для обеспечения безопасности в «Интернете вещей» // Компьютерная оптика. 2019. № 43 (1). C. 137-154.

13 Bukht R., Heeks R. Defining, conceptualising and measuring the digital economy // Development Informatics working paper. 2017. № 68.

14 Popkova E.G. Digital Economy: Complexity and Variety vs. Rationality. Springer International Publishing AG, 2019.

15 Litvinenko V. S. Digital economy as a factor in the technological development of the mineral sector // Natural Resources Research. 2020. V. 29. № 3. P. 1521-1541.

\section{References}

1 Ovchinnikov V.N., Ketova N.P. System-Complementary effect of interaction of innovative potential and institutional environment of the region. Regional economy. 2016. vol. 12. no. 2. pp. 537-546. doi: 10.17059/2016-2-18 (in Russian).

2 Timakova R.T., Maiseyenko A.V., Iliukhin R.V. Modern Aspects of Managing the Institutional Environment of Higher Education. Proceedings of the International Scientific Conference «Far East Con» (ISCFEC 2020). vol. 128. pp.1104-1112. doi:10.2991/aebmr.k. 200312.152

3 Timakova R.T., Iliukhina Iu. V. Features of the open educational space of the Russian Federation and the Republic of Belarus in the conditions of digitalization. I international scientific and practical conference "Trends in the development of e-education in Russia and abroad".2020. pp. 170-173. (in Russian).

4 Golovko O.I. Organizational and economic mechanism for ensuring innovative development of organizations. Economy and management of the national economy. 2016. no. 4 (137). pp. 92-94. (in Russian).

5 Zhang M.L., Chen M.S. China's digital economy: Opportunities and risks. International Monetary Fund, 2019.

6 Kregel D.A. State-based mechanisms of stimulation of innovative activities in railway transport. Journal of the University. 2018. no. 2. pp. 57-63. (in Russian).

7 Bespalova I.V. Transformation of the innovation model in modern conditions of the economy structure. Fundamental study. 2015. no. 12 (2). pp. 346-355. (in Russian).

8 Watanabe C. et al. Measuring GDP in the digital economy: Increasing dependence on uncaptured GDP. Technological Forecasting and Social Change. 2018. vol. 137. pp. 226-240.

9 Eleodor D. Big tech, big competition problem? Quality-Access to Success. 2019. vol. 20 (S3). pp. 49-57. URL: https://search.proquest.com/openview/58271826c315d0334b7e9cc6e79a756a/1.pdf? pq-origsite=gscholar\&cbl=1046413

10 Kuladzhi T., Babkin A., Murtazaev S.A. Matrix tool for efficiency assessment of production of building materials and constructions in the digital economy. Advances in intelligent systems and computing. 2017. vol. 692. pp. 1333-1346. doi: 10.1007/978-3-319-70987-1_141

11 Blaginin V., Ergunova O., Pyankova S. Automobile transport infrastructure in the system of maintaining social well-being. Conference Title Abbreviation \& a Year Conference Title. 2018. pp. 62-70.

12 Evsyutin O.O., Kokurina A.S., Meshcheryakov R.V. Review of methods for embedding information in digital objects to ensure security in the Internet of things. Computer optics. 2019. no. 43 (1). pp. 137-154. (in Russian).

13 Bukht R., Heeks R. Defining, conceptualising and measuring the digital economy. Development Informatics working paper. 2017. no. 68.

14 Popkova E.G. Digital Economy: Complexity and Variety vs. Rationality. Springer International Publishing AG, 2019.

15 Litvinenko V.S. Digital economy as a factor in the technological development of the mineral sector. Natural Resources Research. 2020. vol. 29. no. 3. pp. 1521-1541.

\section{Сведения об авторах}

Роза Т. Тимакова докторант, к.с.-х.н., доцент, кафедра туристического бизнеса и гостеприимства, Уральский государственный экономический университет, ул. 8 Марта/Народной воли, 62/45, г. Екатеринбург, 620144, Россия, trt64@ mail.ru (iDhttps://orcid.org/0000-0002-4777-1465

Ольга Т. Ергунова к.э.н., доцент, зав. кафедрой, кафедра туристического бизнеса и гостеприимства, Уральский государственный экономический университет, ул. 8 Марта/Народной воли, 62/45, г. Екатеринбург, 620144, Россия, ergunova-olga@ya.ru

(D)https://orcid.org/0000-0002-1714-7784

\section{Вклад авторов}

Все авторы в равной степени принимали участие в написании рукописи и несут ответственность за плагиат

\section{Information about authors}

Roza T. Timakova doctoral candidate, Cand. Sci. (Agric.), tourism business and hospitality department, Ural State Economic University, St. 8-e Marta/Narodnoy voli, 62/45, Ekaterinburg, 620144, Russia , trt64@mail.ru

(iDhttps://orcid.org/0000-0002-4777-1465

Olga T. Ergunova Cand. Sci. (Econ.), associate professor, head of department, tourism business and hospitality department, Ural State Economic University, St. 8 Marta/Narodnoy voli, 62/45, Ekaterinburg, 620144, Russia, ergunova-olga@ ya.ru

(Dhttps://orcid.org/0000-0002-1714-7784

\section{Contribution}

All authors are equally involved in the writing of the manuscript and are responsible for plagiarism

\author{
Conflict of interest
}

The authors declare no conflict of interest.

\begin{tabular}{ccc} 
Поступила 12/10/2020 & После редакции 28/10/2020 & Принята в печать 11/11/202 \\
\hline Received 12/10/2020 & Accepted in revised 28/10/2020 & Accepted 11/11/2020
\end{tabular}

